\title{
¿Y si nos preocupamos del ejercicio profesional en todas las asignaturas de la carrera de Derecho, inclusive en las teóricas? Estudio y puesta en práctica de una propuesta metodológica que mejore el desempeño de los estudiantes con énfasis en la abogacía
}

\author{
E se nos preocupássemos com a prática profissional em todas as disciplinas da \\ faculdade de Direito, inclusive as teóricas? Estudo e implementação de uma proposta \\ metodológica que melhore o desempenho discente com ênfase na advocacia
}

\section{What if we worry about professional practice in all subjects of the Law School, including the theoretical ones? Study and implementation of a methodological proposal that improves student performance with an emphasis on the legal profession}

\author{
Juan Pablo Díaz Fuenzalida \\ Universidad Autónoma de Chile
}

\begin{abstract}
RESUMEN En los últimos años, ha habido una especial preocupación por el desempeño práctico de los futuros abogados. Las universidades han hecho modificaciones curriculares integrando cursos donde predomina lo práctico, pero manteniendo asignaturas teóricas. Con esto, solo se aliviaría en parte la problemática, salvo que en las asignaturas se incorporaran metodologías y técnicas guiadas al ejercicio de la profesión. Así, se plantea que es posible mejorar el desempeño de los estudiantes con una propuesta metodológica que dé énfasis en la abogacía, tanto en asignaturas tradicionalmente teóricas como en las prácticas. Esto, dado que se desarrollarían competencias necesarias para aplicar las ciencias jurídicas, lo que se debe evidenciar con productos propios del ejercicio profesional en sus variados ámbitos de acción. Para realizar el ejercicio de comprobación, la muestra consideró asignaturas de cursos de derecho sustantivo y adjetivo, de jornada diurna y vespertina, y de campus diferentes. El resultado ha evidenciado que el planteamiento es correcto. Es posible aplicar la metodología, destacando que mejora una serie de desempeños, tanto en estudiantes como en profesores. No se divisan diferencias significativas entre naturaleza de asignatura, campus ni jornada, pero sí sobre el tipo de metodología pedagógica empleada.
\end{abstract}


PALABRAS CLAVE Asignaturas teóricas, ejercicio de la profesión, abogacía, productos de aprendizaje, instrumentos de evaluación.

RESUMO Nos últimos anos, tem havido uma preocupação particular com o desempenho prático dos futuros advogados. As universidades têm feito modificações curriculares integrando cursos onde predominam as questões práticas, mas mantendo as disciplinas teóricas. Por isso, o problema seria apenas parcialmente amenizado, a menos que incorporem metodologias e técnicas pautadas no exercício da profissão. Assim, propõe-se que seja possível melhorar o desempenho dos alunos com uma proposta metodológica que valorize a profissão jurídica, tanto nas disciplinas tradicionalmente teóricas quanto nas práticas. Isso, visto que seriam desenvolvidas as competências necessárias para a aplicação das ciências jurídicas, o que deve ser comprovado com produtos da prática profissional em seus diversos campos de atuação. Para a realização do exercício de verificação, a amostra considerou disciplinas de cursos de direito substantivo e adjetivo, diurnos e noturnos, e de campus diferentes. O resultado mostrou que a abordagem está correta. É possível aplicar a metodologia, destacando que ela melhora uma série de desempenhos, tanto de alunos quanto de professores. Não existem diferenças significativas entre a natureza da matéria, o campus ou o dia, mas existem diferenças no tipo de metodologia pedagógica utilizada.

PALABRAS-CHAVE Disciplinas teóricas, exercício da profissão, direito, produtos de aprendizagem, instrumentos de avaliação.

ABSTRACT In recent years there has been a concern for the practical performance of future lawyers. Universities have made curricular modifications integrating courses where practical matters predominate but maintaining theoretical subjects. For this reason, the problem would only be partially alleviated, unless they incorporate methodologies and techniques guided by the exercise of the profession. Thus, it is proposed that it is possible to improve student performance with a methodological proposal that emphasizes the legal profession, both in traditionally theoretical and practical subjects. This, given that the necessary competencies would be developed to apply legal sciences, which must be evidenced with products of professional practice in its various fields of action. To carry out the verification exercise, the sample considered subjects from substantive and adjective law courses, day, and evening hours, and from different campuses. The result has shown that the approach is correct. It is possible to apply the methodology, highlighting that it improves a series of performances, both in students and teachers. There are no significant differences between the nature of the subject, the campus, or the day, but there are differences in the type of pedagogical methodology used.

KEYWORDS Theoretical courses, practice of the profession, advocacy, learning products, evaluation instruments. 


\section{Planteamiento del problema}

\section{Preocupación por el desempeño práctico de los nuevos abogados}

En los últimos años, ha habido una fuerte preocupación por el desempeño de los nuevos egresados de la carrera de Derecho por parte de distintos sectores. Esto, especialmente desde la Corporación de Asistencia Judicial, que ha llamado la atención, entre otras cosas, sobre los aspectos prácticos necesarios para el ejercicio profesional de la abogacía. ${ }^{1}$ Asimismo, en 2018, el director de la Academia Judicial ha referido que la calidad de los abogados ha ido descendiendo en los últimos años, acentuando la crítica y la responsabilidad en las escuelas de Derecho. ${ }^{2}$ Por cierto, es un asunto que ha estado latente hace poco más de 10 años. Así, el ex presidente de la Corte Suprema, Milton Juica, afirmó que entregan el título «sin tener conciencia clara y cierta» de si quien lo recibe está capacitado efectivamente para desempeñar la profesión. ${ }^{3}$ Ante ello, es menester tomar medidas, pensar sobre qué está ocurriendo y en cómo poder mejorar la situación.

Con la garantía constitucional de libertad de enseñanza, consagrada en el artículo 19, número 11, de la Constitución Política, es posible impartir la carrera de Derecho con un gran margen en la elaboración del perfil de egreso de los futuros abogados. Pero no se debe olvidar la esencia de estos, que es ser competente para litigar en tribunales de justicia. En efecto, en Chile es posible estudiar derecho en 43 universidades, ${ }^{4}$ cada una con un perfil, plan de estudios y competencias distintas. Sin embargo, no debemos obviar al artículo 520 del Código Orgánico de Tribunales, que define a los abogados como aquellas «personas revestidas por la autoridad competente de la facultad de defender ante los tribunales de justicia los derechos de las partes litigantes». De esta manera, la formación en litigación es sencillamente el cumplimiento de la legislación chilena. Esto, sin perjuicio de que, adicionalmente, la abogacía es más variada aún que el enfoque restrictivo del artículo citado. Así, Matus (2007) nos recuerda otros ámbitos de aplicación del ejercicio profesional, adicional al actuar en tribunales como litigante, también como funcionario público, miembro de una organización empresarial o profesional, en la administración de justicia, pudiendo agregar, en cierta medida, a los abogados que se especializan en lo académico, los

1. Gabriela Pizarro, «Juicio a las escuelas de derecho: Las alarmantes falencias de los nuevos abogados», Ciper, 2 de marzo de 2016, disponible en bit.ly/3fokYuo.

2. P. Campos y C. Carvajal, «¿Eunacom para abogados? Las críticas a la formación profesional». Diario Uchile, 1 de mayo de 2018, disponible en bit.ly/30N2bbd.

3. «Corte Suprema: No podemos verificar la calidad de los abogados recién egresados», Emol, 3 de marzo de 2009, bit.ly/3fDflhn.

4. Juan José, «Derecho en Chile: Dónde estudiar y mejores universidades», Cursando.cl, disponible en bit.ly/3foHRgR. 
juristas, y a los asesores legales, entre otras amplias actividades relacionadas con las ciencias jurídicas.

No obstante, es menester sumar a lo expuesto lo indicado por la Comisión Nacional de Acreditación (CNA), relativo a los criterios de evaluación para las carreras de Derecho. Esto, dado que su objetivo es verificar y promover la calidad de las instituciones de educación superior en Chile (Suazo, 2017). Esto significa, para los directores de la carrera, docentes y estudiantes, considerar, en los procesos de enseñanza-aprendizaje, la vertiente del desarrollo profesional. Así, la CNA (2020) ha manifestado que «el currículo conducente a la formación del Licenciado en Ciencias Jurídicas debe comprender todos los aspectos esenciales de la disciplina y conferir una formación tanto general como científica necesaria para el adecuado desempeño profesional». Es decir, lo teórico del derecho debe poder aplicarse en el ejercicio de la abogacía, entendiéndose en su sentido más restrictivo (como lo es la litigación), pero también al amplio campo de su desarrollo (como el ejercicio de juez, notario, funcionario de la Administración del Estado y asesor legal, entre otros). Lo relevante es que dicho ejercicio pueda materializarse en productos, evidencias tanto de aprendizaje en la carrera de Derecho como de ejercicio efectivo de la profesión (un juez con una sentencia, un asesor con un informe en derecho o un litigante con una demanda o recurso, por ejemplo).

Todo esto, nos podría llevar a preguntarnos: ¿Más o menos teoría? ¿Más o menos práctica? Sin embargo, haría falta una premisa aún más relevante: ¿Cómo lo teórico es trasladable a lo práctico? Este es el verdadero dilema que debemos resolver quienes dedicamos gran parte de nuestro tiempo a las facultades de Derecho. Estos puntos que inician la investigación son relevantes, ya que el conocimiento científico se produce en un contexto histórico y cultural (Gómez y Rubio, 2017). Todo esto, es parte de lo que motiva a generar una propuesta metodológica que mejore el desempeño de los estudiantes con énfasis en la actividad de la abogacía.

\section{Modificaciones de los planes de estudio hacia lo práctico}

La enseñanza del derecho ha sido un punto de relevancia en las facultades más prestigiosas a nivel mundial. En el caso de Estados Unidos, existe abundancia en literatura no escrita por pedagogos a secas, en que se une a actores clave en el ejercicio profesional. Se ha calificado como un debate animado por las asociaciones de abogados que demandan más práctica, en relación con el carácter predominantemente teórico de la enseñanza en las escuelas de Derecho (Martínez y Arufe, 2015). Lo mismo ocurre en el caso europeo, en que desde 1999 comenzó a desarrollarse un cambio de paradigma en la educación superior, conformado por tres pilares fundamentales: la movilidad, la competitividad internacional y la empleabilidad (Orihuela y Rubio, 2010). Es este tercer elemento el que repercute en el ejercicio profesional de los futuros abogados. 
De él, es necesario preocuparse en y durante la carrera de Derecho, y no al final en la Corporación de Asistencia Judicial o en cualquier otra alternativa de práctica profesional. Para el caso chileno, es aún más relevante, ya que es posible litigar estando matriculado en una escuela de Derecho en tercer año de la carrera según lo dispone la Ley 18.120. Aunque necesite del patrocinio de un abogado, claramente quien reali$\mathrm{za}$ actuaciones procesales es el procurador, que puede ser un estudiante.

Por esto, es relevante revisar los planes de estudios, conocidos comúnmente como mallas curriculares. En un estudio publicado en 2012, se sintetizan estos a propósito del modelo chileno, que es temporalmente extenso y dogmáticamente muy exhaustivo (Solari, 2012). Si uno hace memoria de cuando cursó la carrera de Derecho, efectivamente había pocas asignaturas prácticas. Recuerdo, por ejemplo, Derecho Procesal unido a Taller de Litigación y, especialmente, Clínica Jurídica, que era justamente una de aquellas materias catalogadas de atención directa, ya que trabaja resolviendo casos reales (Bonet, 2018a), en que los más beneficiados eran el patrocinado y el alumnado (Bonet, 2018b). No puedo estar más de acuerdo con ello: los estudiantes teníamos un aprendizaje significativo, poniendo en práctica lo aprendido y, por ello, se llegaba de mejor forma a enfrentar a la masividad de causas que se debe tramitar de forma casi inmediata al ingreso en la Corporación de Asistencia Judicial. Es más, no solo generaba competencias prácticas, sino que reafirmaba la misión social que tienen las universidades y particularmente las clínicas jurídicas (Blázquez, 2015), además del compromiso con la comunidad.

Por cierto, hay muchos casos en que lo anterior ha ido variando. En efecto, cada vez hay más asignaturas prácticas. Solo por dar un ejemplo, en la carrera de Derecho de la Universidad Autónoma de Chile (2020) se considera, en el segundo semestre, Argumentación Jurídica y Debate; en el tercer semestre, Negociación y Ética Profesional; en el quinto semestre, Redacción Forense; en el séptimo semestre, Solución Alternativa de Conflictos; en el octavo semestre, Taller de Litigación; en el noveno semestre, Clínica Jurídica; y, en el décimo semestre, Taller de Litigación Avanzada. En el caso de la Universidad de Chile (2020), la malla curricular incorpora, en el cuarto semestre, un optativo, Solución Alternativa de Controversias; en séptimo semestre, Clínica (obligatoria); en el octavo semestre, Profesión Jurídica; en el noveno semestre, Clínica (optativa) y, además, un Taller I; y, en el décimo semestre, Taller II. Revisando la malla curricular de la carrera de Derecho de la Universidad Católica de Chile, encontramos, en el primer semestre, la asignatura de Desarrollo de Habilidades Comunicativas de los Abogados y Taller de Metodología de la Investigación; en el noveno semestre, Clínica Jurídica I; y en el décimo semestre, Clínica Jurídica II. Es decir, también se sigue la lógica de incluir asignaturas relacionadas con el ejercicio profesional, guiadas a aprendizajes prácticos, aunque son una minoría en el plan de estudios y pueden generar la idea de dividir lo teórico de lo práctico.

De esta manera, se ha hecho la tarea en las nuevas asignaturas en relación con 
la problemática planteada al inicio, pero ¿qué ocurre en las demás? Efectivamente, no todo puede ser un taller, también son necesarios los conocimientos teóricos para que estos puedan ser aplicados. Para esto, hay diversas metodologías para cumplir con la enseñanza-aprendizaje de los saberes. Así, la criticada clase magistral tiene sus puntos positivos y negativos. Es más, si se le aplican los principios y técnicas de la didáctica actual, puede transformarse de una clase meramente expositiva a una trascendente o formativa (Elgueta y Palma, 2014). En efecto, las ventajas de la clase magistral son el ahorro de tiempo y de medios, la presencia del profesor, la atención de grupos numerosos, la facilitación de mucha información elaborada, y la vitalización de las ideas y de los hechos que aparecen en los manuales, haciéndolos más accesibles a los alumnos. Las desventajas serían que se habla a una audiencia anónima, la poca participación del alumnado, la dificultad en la retroalimentación, que no se atiende al trabajo autónomo de los estudiantes y que se genera el peligro de que se generalice el hábito de estudiar únicamente mediante notas tomadas en clase (De la Peña y Marcos, 2010). Con ello, podríamos tener cubierto, al menos de momento, el conocimiento teórico. Sin embargo, haría falta la aplicación.

\section{No solo es suficiente innovar en metodologías de aprendizaje, es necesario materializar hacia el ejercicio profesional}

En efecto, es posible adquirir lo práctico a través de la metodología de casos. Por cierto, no se debe confundir con las técnicas en que se buscan o determinan criterios jurisprudenciales, como es tradicional en Estados Unidos, que se basa en un ordenamiento jurídico centrado en la revisión judicial (Gabilondo, 2017). Así, los abogados se enfrentan, en cada momento, a situaciones de hecho por solucionar, es decir, deben resolver casos, por lo que cuesta dilucidar una mejor opción. Sin embargo, de acuerdo con la situación y la materia del derecho, la solución del caso puede revestir distintas alternativas. Así, en Derecho Civil, si la asignatura trata de «contratos», se podrá redactar una demanda que exija el cumplimiento forzado de una obligación o la elaboración de un contrato. En Derecho Constitucional, redactar un recurso de protección o elaborar un informe en derecho sobre la constitucionalidad de una ley. Con casos prácticos, no solo enfocados necesariamente a litigación, los estudiantes se ven obligados a trabajar, en conjunto con sus compañeros, para resolver problemas complejos y auténticos que les sirven para desarrollar tanto sus conocimientos teóricos como prácticos; tienen que manejar plataformas informáticas recomendadas por el docente para buscar jurisprudencia o doctrina especializada; y, además, utilizar tiempo fuera del aula (Selma, 2010), que sirven para la realización efectiva de los créditos de trabajo autónomo.

Sin embargo, la metodología utilizada y puesta en marcha tuvo presente aspectos de lo que se conoce como Aprendizaje Basado en Problemas (ABP). En efecto, se 
plantea un problema o caso a los estudiantes y estos, en grupo (prefiero hablarles de equipo), deben seguir una serie de premisas para llegar a una solución. Se toma en cuenta este método por sus puntos positivos, ya que destaca, entre otros, la habilidad para resolver problemas reales (Ramírez, 2017). Asimismo, se fomentan una serie de competencias relacionadas en la vinculación de los conocimientos teóricos con la práctica, como lo es la resolución de problemas o el desarrollo y ejecución de proyectos (Gil-Galván, 2018). Pero no es suficiente un ABP, ya que hay que añadir el enfoque profesional. Es decir, no es solo llegar a una mejor decisión, hay que ponerla en práctica a través de productos que se elaboran en el ejercicio de la abogacía, como una demanda, un recurso, un contrato, un testamento o un informe en derecho, entre otros.

Es, en otras palabras, acercar la realidad a los estudiantes. Lo que se busca es generar competencias para analizar, interpretar y aplicar las normas jurídicas. Es relevante considerar el desarrollo de la creatividad, es decir, no es solo aplicar, sino que es necesario generar un ambiente guiado a la búsqueda de respuestas apropiadas por parte de los estudiantes, con un enfoque hacia el mundo profesional (Camilloni, 2019). Los profesores de derecho tenemos la difícil misión de preparar para un mundo desconocido. La respuesta puede ser la creatividad para obtener un mejor índice de adaptabilidad. Pero, como se ha dicho, es una creatividad que está guiada hacia lo profesional. Inclusive, hay experiencias hasta en el tradicional examen de grado de la carrera de Derecho en Chile, que cada día se está enfocando más en competencias que en los contenidos propiamente tales. Como, por ejemplo, pudiendo evaluar la redacción de textos jurídicos, el razonamiento, la argumentación jurídica y la interpretación del derecho (Beca y otros, 2019). Es decir, competencias de ejercicio de la abogacía, ya sea como litigante o en otras esferas de la profesión.

En ese sentido, es importante tener en cuenta que, desde los primeros años de la carrera, se deben comenzar a desarrollar las competencias de ejercicio profesional. En efecto, este tipo de habilidades van evolucionando, retroalimentándose, mejorando a través del tiempo. $\mathrm{Si}$, por ejemplo, exigimos redacción jurídica, lo primero es que los estudiantes puedan «redactar». Experiencias curriculares hay al respecto, que contemplen denominadas competencias basales (Sologuren y otros, 2019), para el desarrollo inicial en nuestra disciplina. El ejercicio de la abogacía es una actividad y, como tal, se debe empezar a practicar desde los primeros años. De antaño, se veía a las escuelas de derecho como lugares donde se adquirían datos y, como mucho, lo práctico a través de una pasantía. Gran falacia, ya que, en esta etapa, el pasante, no en muy pocas ocasiones era autónomo, más bien, en palabras de De Miranda (2017), habiendo un gran riesgo de convertirse en un «ver hacer», lo que es formativo, pero no es suficiente para «hacer por sí mismos». De ahí, la importancia de comenzar en los primeros años a formar no solo en saberes, sino que también en saber hacer, pero con enfoque en una actividad, que es el ejercicio profesional. 
Ahora bien, la solución del caso no es suficiente si no se materializa en un producto que evidencie el aprendizaje. En este sentido, ayuda a la propuesta metodológica el aprendizaje clínico, entendido como aquel aprendizaje que tiene lugar haciendo aquello que precisamente hacen los abogados. O también, a cualquier tipo de educación que use métodos interactivos para enseñar habilidades prácticas del ejercicio del derecho, lawyering skills (Orihuela y Rubio, 2010). Así, no se debe confundir, necesariamente, con metodologías de aprendizaje más servicio conocidas como $\mathrm{A}+\mathrm{S}$, que unen trabajo, servicio y aprendizaje (Barrios y otros, 2012). Esto es lo que verdaderamente hacen las Clínicas Jurídicas que atienden a personas para presentar defensas en tribunales de justicia, asesorías, entre otras. Sin entrar en mayores análisis, si bien es interesante la metodología, implica una mayor cantidad de recursos (módulos de atención, profesores asistentes, ayudantes e infraestructura) y de asunción de responsabilidades con los usuarios, lo que tornaría complejo un $\mathrm{A}+\mathrm{S}$ en todas las asignaturas de la carrera.

Con todo, tomando lo positivo de la clase magistral, del método de casos, del estudio de jurisprudencia y del aprendizaje clínico (no entendido necesariamente como $A+S$ ), es posible elaborar una propuesta académica que esté guiada al aprendizaje de lo teórico llevado a lo práctico. Es así como se planificaron y se materializaron satisfactoriamente, en 2018, distintas asignaturas teóricas y prácticas en la carrera de Derecho de la Universidad Autónoma de Chile. Estas dieron cuenta de una propuesta metodológica enfocada al ejercicio de la abogacía.

\section{Hipótesis y metodología}

En este momento, es coherente elaborar una hipótesis de investigación, ya que con ella se podrán obtener conclusiones en sentido positivo o negativo, siendo cualquiera de los dos resultados un avance en términos científicos (Espinoza, 2018). Así, de acuerdo con lo expuesto, y especialmente en relación con la problemática planteada, se presenta como hipótesis a comprobar que es posible mejorar el desempeño de los estudiantes con una propuesta metodológica que dé énfasis en la actividad de la abogacía. Ello, tanto en asignaturas tradicionalmente teóricas como en las prácticas. Así, se desarrollan competencias necesarias para aplicar las ciencias jurídicas, lo que se evidencia con productos propios del ejercicio profesional.

Para lograrlo, es menester considerar, en el proceso, una serie de aspectos docentes relacionados con la implementación de instrumentos de evaluación. Existe una variedad de formatos (Vallés y otros, 2018), pero lo importante, valga la redundancia, es que evalúen desempeños materializados en productos académicos que sean los mismos que se utilizan en el ejercicio de la abogacía.

Para la comprobación de la hipótesis, para la investigación se utilizó el paradigma analítico-explicativo. Por esto, el diseño a emplear es explicativo, cuantitativo, deduc- 
Tabla 1. Muestra para implementación de propuesta metodológica guiada a la actividad de la abogacía

\begin{tabular}{|llllll|}
\hline Número & Asignatura & Sede & Campus & Jornada & Estudiantes \\
\hline 1 & Teoría del Proceso & Santiago & El Llano & Vespertino & 55 \\
\hline 3 & Teoría del Proceso & Santiago & El Llano & Diurno & 49 \\
\hline 4 & Redacción Forense & Santiago & Providencia & Vespertino & 19 \\
\hline 5 & Derechos y Garantías Constitucionales & Santiago & Providencia & Diurno & 44 \\
6 & Fuentes de las Obligaciones & Santiago & Providencia & Diurno & 37 \\
\hline Total de estudiantes para la muestra & Santiago & Providencia & Diurno & 9 \\
\hline
\end{tabular}

tivo y transversal, lo que se llevó a cabo a través de una base investigativa de carácter experimental (Hernández y otros, 2013). La implementación de la propuesta metodológica guiada a la actividad de la abogacía de este trabajo tomó como muestra a seis cursos, que van desde segundo año de derecho hasta quinto. Se consideraron secciones de jornada diurna y vespertina de la sede de Santiago, del campus Providencia y de El Llano Subercaseaux. Todo esto, para que la muestra fuese representativa (Manterola y Otzen, 2013), tanto desde el punto de vista de los estudiantes como de la naturaleza de las asignaturas a evaluar. En efecto, los cursos se pueden apreciar en la tabla 1.

Se trabajó con distintos profesores para poner en práctica la implementación de la propuesta. Además, se consideró una etapa fundamental, que fue la de validación de los instrumentos de evaluación y de casos a desarrollar por los estudiantes. Esto se hizo con una pauta de cotejo y a partir del desempeño de los estudiantes por medio de una rúbrica. Otra etapa significativa fue aquella de retroalimentación a los estudiantes, especialmente si era la primera vez que hacían un escrito o algún producto de la abogacía que pusiera en práctica sus saberes adquiridos.

Se acordó que, al menos en una evaluación, debía aplicarse la metodología presentada, que fue concretamente la prueba regular 3 o nota 3 (en la reglamentación actual, son en total cuatro evaluaciones de 30\%, 30\%, 30\% y 10\%). Así, por ejemplo, en Derechos y Garantías Constitucionales, la evaluación 1 fue una prueba escrita (formato tradicional), la evaluación 2 fue una prueba solemne oral (tipo examen oral tradicional en derecho) y la evaluación 3 fue la solución de un caso a través de la elaboración de un recurso de protección (propuesta metodológica que aborda el presente trabajo). Así, igualmente con las demás, en Fuentes de las Obligaciones se debía realizar asesoría materializada en un informe más una escritura, y en Derecho Civil IV redactar un testamento. Es decir, en asignaturas tradicionalmente teóricas, se implementa una metodología guiada al ejercicio práctico de la abogacía, tanto en el sentido restrictivo como amplio de la profesión. 
Posteriormente, se evaluó la propuesta metodológica con la percepción de los agentes clave, a saber, estudiantes y profesores que participaron. Asimismo, se examinaron los resultados del desempeño de los estudiantes, generando una comparación con las otras evaluaciones tradicionales (nota 1 y 2). Se han tomado las medidas pertinentes para mantener el anonimato y el resguardo de los datos personales de los participantes.

En adelante, se presentarán los resultados de la investigación con su respectivo análisis.

\section{Presentación de los resultados y su análisis}

Percepción de los agentes clave: Los docentes

Es relevante evaluar el proceso y la metodología utilizada. Así, se consultó a los docentes que participaron del proyecto y a los estudiantes. Los resultados fueron muy clarificadores.

En relación con el aprendizaje de los estudiantes, los docentes de las áreas de derecho procesal y de derecho civil respondieron lo que se presenta en la tabla 2 (no se incluyó al docente de derecho constitucional por estar a cargo de la propuesta metodológica, ya que sería equivalente a ser juez y parte al mismo tiempo).

De los resultados, podemos destacar que se establecieron las directrices de la implementación del proyecto en forma clara y precisa, dejando la libertad necesaria para que cada docente pudiera desarrollar su cátedra de acuerdo con la pericia o la experiencia de su enseñanza-aprendizaje.

Por parte de los docentes, se consideró como un método que efectivamente logró que los estudiantes aprendieran a aplicar el contenido visto en clases y a materializarlo con un instrumento de evaluación completamente funcional. Además, agregaron que la metodología utilizada acercó a los estudiantes al ejercicio de la profesión.

Se concluyó que es recomendable que se siga aplicando la metodología propuesta, no solo en las cátedras respectivas, sino que en las demás.

\section{Percepción de los agentes clave: Los estudiantes}

Para esta sección, se consultó a estudiantes de forma individual y fueron seleccionados aleatoriamente a partir del Campus Providencia y de El Llano. Los resultados se pueden ver en la tabla 3 .

Destaca que los estudiantes han recibido muy bien la iniciativa, considerándola como innovadora y muy útil para el ejercicio de la profesión, y que han afrontado la evaluación como una oportunidad para aplicar sus conocimientos, superando los nervios iniciales de estar ante una distinta a las anteriores. 
Tabla 2. Percepción de los docentes de las áreas de derecho procesal y de derecho civil

\begin{tabular}{|c|c|c|c|}
\hline Núm. & Pregunta & Respuesta del profesor de Derecho Procesal & Respuesta del profesor de Derecho C \\
\hline 1 & $\begin{array}{l}\text { ¿Qué producto instó a que hicieran } \\
\text { los estudiantes demanda, con- } \\
\text { testación, recurso de protección, } \\
\text { testamento u otro, y en que } \\
\text { asignatura? }\end{array}$ & $\begin{array}{l}\text { Frente a una demanda, redactar un instru- } \\
\text { mento aplicando la materia vista en clases, } \\
\text { contestación, demanda reconvencional, } \\
\text { excepciones dilatorias y perentorias. }\end{array}$ & $\begin{array}{l}\text { Ellos tuvieron que hacer análisis } \\
\text { de casos evaluados a través de una } \\
\text { rúbrica. Realizaron, en derecho suce- } \\
\text { sorio, un testamento, relacionando } \\
\text { los regímenes matrimoniales y, en } \\
\text { fuente de las obligaciones, realizaron } \\
\text { contratos, promesa, compraventa y } \\
\text { mandato, los más fuertes. }\end{array}$ \\
\hline 2 & $\begin{array}{l}\text { ¿El producto que se solicita es } \\
\text { necesario en el ejercicio de la } \\
\text { abogacía? }\end{array}$ & Absolutamente. & Sí. \\
\hline 3 & $\begin{array}{l}\text { ¿Cuál es su opinión con respecto } \\
\text { a la implementación de esta } \\
\text { modalidad de aprendizaje? }\end{array}$ & $\begin{array}{l}\text { Creo que es un modelo que tiene que } \\
\text { ver mucho con los objetivos que busca la } \\
\text { universidad, centrado en el alumno y no en } \\
\text { el docente, aprendiendo, haciendo desde } \\
\text { la práctica más que en la teoría. }\end{array}$ & $\begin{array}{l}\text { Bien ordenada, adecuada, bien } \\
\text { coordinada, los profesores tuvimos un } \\
\text { margen de libertad para implementar } \\
\text { el proyecto según la expertis de } \\
\text { cada uno. Me parece bien entregar } \\
\text { directrices de cómo hacer las cosas y } \\
\text { las libertades para ejecutarlas. }\end{array}$ \\
\hline 4 & $\begin{array}{l}\text { ¿Cómo percibió la respuesta de los } \\
\text { estudiantes ante esta modalidad } \\
\text { de evaluación? }\end{array}$ & $\begin{array}{l}\text { Bien, entendieron el objetivo de la evalua- } \\
\text { ción, que es la aplicación del conocimiento. }\end{array}$ & $\begin{array}{l}\text { Bastante buena, les gustó, hice } \\
\text { algunos ejercicios preliminares. } \\
\text { Después, fueron tomando el ritmo y } \\
\text { se obtuvieron buenos resultados. }\end{array}$ \\
\hline 5 & $\begin{array}{l}\text { ¿Los estudiantes obtuvieron los } \\
\text { aprendizajes esperados relaciona- } \\
\text { dos con el producto a evaluar? }\end{array}$ & $\begin{array}{l}\text { Sí, efectivamente, algunos mejor que } \\
\text { otros, pero sí aprendieron. }\end{array}$ & Sí. \\
\hline 6 & $\begin{array}{l}\text { ¿Cómo logró buenos resultados en } \\
\text { los estudiantes y sus productos a } \\
\text { evaluar, hizo retroalimentación, } \\
\text { dio casos? }\end{array}$ & $\begin{array}{l}\text { Antes de implementar esto, en mis clases } \\
\text { había aplicación práctica de la materia en } \\
\text { clase, entonces tenían nociones. Además, } \\
\text { las rubricas dieron las instrucciones nece- } \\
\text { sarias con los objetivos de la evaluación, } \\
\text { que ayudaron a la implementación. }\end{array}$ & $\begin{array}{l}\text { Bastante buena, les gustó, hice } \\
\text { algunos ejercicios preliminares. } \\
\text { Después, fueron tomando el ritmo y } \\
\text { se obtuvieron buenos resultados. }\end{array}$ \\
\hline 7 & $\begin{array}{l}\text { ¿Considera que en su asignatura y } \\
\text { en otras es necesario este tipo de } \\
\text { actividades para obtener abogados } \\
\text { cada vez más competentes en el } \\
\text { ejercicio profesional? }\end{array}$ & $\begin{array}{l}\text { Sí, claro, porque lo normal es que algunos } \\
\text { estudiantes se quejen de que no saben } \\
\text { redactar ni aplicar conocimientos. Con esto, } \\
\text { están aprendiendo a hacerlo. }\end{array}$ & Sí, me parece. \\
\hline 8 & $\begin{array}{l}\text { ¿Los estudiantes tuvieron trabajo } \\
\text { autónomo - extra-aula_- para } \\
\text { elaborar el producto? }\end{array}$ & $\begin{array}{l}\text { Sí, comenzaron en horario de clases, para } \\
\text { acompañarlos y resolver dudas, y tuvieron } \\
\text { tiempo extra para analizar el tema. }\end{array}$ & $\begin{array}{l}\text { Sí, comenzaron en clases y también } \\
\text { les di un espacio fuera de clases para } \\
\text { desarrollarlo. }\end{array}$ \\
\hline 9 & $\begin{array}{l}\text { ¿Algo más que agregar relativo al } \\
\text { aprendizaje de los estudiantes? }\end{array}$ & $\begin{array}{l}\text { Creo que es un medio que se debe } \\
\text { extender. }\end{array}$ & Nada más que agregar. \\
\hline 10 & $\begin{array}{l}\text { ¿Recomendaría seguir reali- } \\
\text { zando evaluaciones según esta } \\
\text { modalidad? }\end{array}$ & Sí, de todas maneras. & Sí \\
\hline
\end{tabular}


Tabla 3. Percepción de estudiantes

\begin{tabular}{|c|c|c|c|}
\hline Núm. & Pregunta & $\begin{array}{l}\text { Respuesta de la estudiante del Campus } \\
\text { El Llano }\end{array}$ & $\begin{array}{l}\text { Respuesta del estudiante de la de } \\
\text { Campus Providencia }\end{array}$ \\
\hline 1 & $\begin{array}{l}\text { ¿Cuál es su opinión con respecto a la } \\
\text { implementación de esta modalidad de } \\
\text { aprendizaje? }\end{array}$ & $\begin{array}{l}\text { Buenísimo, porque lo que hemos apren- } \\
\text { dido en clases se pone en práctica, como } \\
\text { cuando ejerces la profesión. }\end{array}$ & $\begin{array}{l}\text { Excelente iniciativa, me parece } \\
\text { una idea innovadora. }\end{array}$ \\
\hline 2 & $\begin{array}{l}\text { ¿Las instrucciones han sido bien } \\
\text { explicadas? }\end{array}$ & $\begin{array}{l}\text { Más o menos, siempre que pasa de } \\
\text { compañero a compañero uno se puede } \\
\text { confundir. }\end{array}$ & Sí, fueron muy detalladas. \\
\hline 3 & $\begin{array}{l}\text { ¿Crees que ha sido ejecutado en forma } \\
\text { adecuada? }\end{array}$ & $\begin{array}{l}\text { Sí, para ser mi primera vez estoy } \\
\text { satisfecha. }\end{array}$ & Sí, me siento satisfecha. \\
\hline 4 & $\begin{array}{l}\text { ¿Cuál fue su actitud al afrontar esta } \\
\text { evaluación en comparación con los } \\
\text { exámenes anteriores? }\end{array}$ & $\begin{array}{l}\text { Pánico, pero después de entender la eva- } \\
\text { luación estuve más relajada y confiada. }\end{array}$ & $\begin{array}{l}\text { Agradecí la oportunidad de } \\
\text { participar en la evaluación, ya } \\
\text { que pude aplicar el contenido } \\
\text { visto en clases. }\end{array}$ \\
\hline 5 & $\begin{array}{l}\text { ¿Ha sentido que, a través de esta modali- } \\
\text { dad, se aplica el conocimiento adquirido? }\end{array}$ & $\begin{array}{l}\text { Por supuesto, todo lo aprendido en } \\
\text { clases. }\end{array}$ & Sí, por supuesto. \\
\hline 6 & $\begin{array}{l}\text { ¿Qué medios utilizó para la realizar el } \\
\text { instrumento y cuánto tiempo? }\end{array}$ & $\begin{array}{l}\text { Utilicé los apuntes de clases, informa- } \\
\text { ción de internet y me tomó alrededor } \\
\text { de } 8 \text { horas. }\end{array}$ & $\begin{array}{l}\text { Me tomo de tres a cuatro días } \\
\text { utilizando el material visto en } \\
\text { clases. }\end{array}$ \\
\hline 7 & $\begin{array}{l}\text { ¿Siente que la aplicación de estas } \\
\text { evaluaciones promueve el estudio? }\end{array}$ & $\begin{array}{l}\text { Mucho más que los exámenes orales, } \\
\text { porque se aplica todo lo que tienes a } \\
\text { disposición. }\end{array}$ & $\begin{array}{l}\text { Sí, totalmente, ya que promueve } \\
\text { la búsqueda autónoma del } \\
\text { conocimiento. }\end{array}$ \\
\hline 8 & $\begin{array}{l}\text { ¿Siente que ha realizado un ejercicio que } \\
\text { lo acerca al ejercicio de la profesión? }\end{array}$ & $\begin{array}{l}\text { Claro, como dije en la primera respuesta, } \\
\text { fue buenísimo, porque lo que hemos } \\
\text { aprendido en clases se pone en práctica, } \\
\text { como cuando ejerces la profesión. }\end{array}$ & Totalmente de acuerdo. \\
\hline 9 & $\begin{array}{l}\text { ¿Siente que ahora puede realizar este } \\
\text { instrumento con mayor precisión y ser } \\
\text { perfectamente funcional? }\end{array}$ & $\begin{array}{l}\text { Sí, ya lo hicimos, así que puedo enfrentar } \\
\text { con más confianza una segunda vez. }\end{array}$ & $\begin{array}{l}\text { Me siento más capacitada para } \\
\text { realizar este instrumento por } \\
\text { segunda vez. }\end{array}$ \\
\hline 10 & $\begin{array}{l}\text { ¿Crees que has cumplido en forma satis- } \\
\text { factoria el objetivo de la evaluación? }\end{array}$ & Sí. & $\begin{array}{l}\text { Sí, como dije en la tercera res- } \\
\text { puesta, me siento satisfecha. }\end{array}$ \\
\hline 11 & $\begin{array}{l}\text { ¿Recomendaría seguir realizando evalua- } \\
\text { ciones según esta modalidad? }\end{array}$ & $\begin{array}{l}\text { Sí, en todas las cátedras deberían hacer } \\
\text { lo mismo. }\end{array}$ & $\begin{array}{l}\text { Sí, debiese ser transversal a todas } \\
\text { las asignaturas. }\end{array}$ \\
\hline
\end{tabular}

Un punto importante es que se han motivado a una búsqueda personal de conocimiento a través de distintos medios.

Han quedado satisfechos con los resultados obtenidos y con la convicción de que, cuando se vuelvan a enfrentar ante una evaluación similar o tengan que redactar dicho instrumento fuera de la sala de clases, podrán superar del obstáculo con éxito.

Con respecto a la evaluación docente, se desprende que el proyecto ha servido en la aplicación de instrumentos de evaluación criteriosos, justos y transparentes.

Además, suma que los estudiantes relacionan las actividades evaluadas con el ejercicio de la profesión y de las competencias que tendrán que aplicar en la abogacía. 
Tabla 4. Percepción de estudiantes, respuestas a pregunta abierta y anónima

\begin{tabular}{|c|c|}
\hline Asignatura & $\begin{array}{l}\text { Respuesta del estudiante en relación con el proyecto de docencia/Evaluación del producto que dé } \\
\text { cuenta de los aprendizajes esperados para el ejercicio profesional en la abogacía }\end{array}$ \\
\hline $\begin{array}{l}\text { DER (D) - Derechos y Garantías } \\
\text { Constitucionales }\end{array}$ & Nos prepara para cada situación luego de la titulación. \\
\hline $\begin{array}{l}\text { DER (D) - Derecho Adminis- } \\
\text { trativo }\end{array}$ & $\begin{array}{l}\text { Debido al gran conocimiento del profesor y sus elevados estudios de magíster y doctorado en el } \\
\text { extranjero, ha desarrollado varias técnicas que hacen su clase más práctica. }\end{array}$ \\
\hline $\begin{array}{l}\text { DER (D) - Derechos y Garantías } \\
\text { Constitucionales }\end{array}$ & $\begin{array}{l}\text { Muy buen docente, siempre dispuesto a ayudar a los alumnos para que sean buenos profesio- } \\
\text { nales. }\end{array}$ \\
\hline $\begin{array}{l}\text { DER (D) - Derechos y Garantías } \\
\text { Constitucionales }\end{array}$ & Entrega herramientas clave para la comprensión de la jurisprudencia y su desarrollo. \\
\hline $\begin{array}{l}\text { DER (D) - Derechos y Garantías } \\
\text { Constitucionales }\end{array}$ & $\begin{array}{l}\text { Se preocupa de hacer justas las evaluaciones, respeta las normas y mantiene la armonía en } \\
\text { clase. }\end{array}$ \\
\hline $\begin{array}{l}\text { DER (D) - Derechos y Garantías } \\
\text { Constitucionales }\end{array}$ & Excelente profesor, dinámico, estructurado, criterioso en evaluar y claro cuando explica. \\
\hline $\begin{array}{l}\text { DER (D) - Derechos y Garantías } \\
\text { Constitucionales }\end{array}$ & $\begin{array}{l}\text { Destacado puesto que integra sentencias y casos reales prácticos para el buen desarrollo del } \\
\text { estudiante. }\end{array}$ \\
\hline DER (V) - Redacción Forense & $\begin{array}{l}\text { Nada que decir. Además, es una excelente profesora y profesional. Explica muy bien e incentiva } \\
\text { la aplicación práctica de todo lo aprendido. También a aprender de nuestros errores. }\end{array}$ \\
\hline DER (V) - Redacción Forense & $\begin{array}{l}\text { Clase didáctica, siempre con miras a que obtengamos la información adecuada y, a la vez, se } \\
\text { busca poner en práctica lo aprendido. }\end{array}$ \\
\hline DER (V) - Teoría del Proceso & $\begin{array}{l}\text { La profesora tiene mucho conocimiento, sus clases son muy entretenidas, fomenta los valores } \\
\text { y el trabajo en equipo. Esto se logró ver en los debates presentados, ya que en un ejercicio } \\
\text { expusieron } 5 \text { compañeros, pero el curso completo estuvo apoyando. Eso lo logró la profesora, } \\
\text { tiene esa energía que irradia y motiva seguir. }\end{array}$ \\
\hline DER (V) - Teoría del Proceso & Prepara la cátedra de una manera fácil, entendible y sus evaluaciones son lo más objetivas. \\
\hline DER (V) - Teoría del Proceso & $\begin{array}{l}\text { Aplica la materia de manera práctica, lo que hace que se comprenda mejor. Muy buena profeso- } \\
\text { ra y preocupada por sus alumnos. }\end{array}$ \\
\hline DER (D) - Teoría del Proceso & $\begin{array}{l}\text { Es una docente que tiene conocimientos amplios y que entrega las herramientas necesarias para } \\
\text { el buen desempeño laboral, es proalumno y siempre tiene disposición para ayudar y resolver } \\
\text { dudas. Su materia es enseñada de forma correcta y, además, lo relaciona con él área en que se } \\
\text { desempeña }\end{array}$ \\
\hline DER (D) - Teoría del Proceso & Profesora completa en todos los aspectos académicos. \\
\hline
\end{tabular}

\section{Percepción de los agentes clave: Respuestas abiertas y anónimas en la evaluación docente}

La universidad utiliza, en el período final de la asignatura, la evaluación docente a través de distintas formas. Una de las más relevantes es aquella en que los estudiantes se dan el tiempo de escribir una respuesta a partir de preguntas abiertas y anónimas. En efecto, en la tabla 4 se evidencian las respuestas relacionadas con la propuesta metodológica empleada. 
Tabla 5. Consolidado del desempeño y de evaluaciones de las asignaturas del proyecto, calificación numérica

\begin{tabular}{|llllllllll} 
Núm. & Asignatura & Campus & Jornada & Nota 1 & Nota 2 & $\begin{array}{l}\text { Nota } \\
\text { proyecto }\end{array}$ & Nota 4 & Nota final \\
\hline 1 & Teoría del Proceso & El Llano & Vespertino & 5,2 & 4,8 & 6,4 & 6,3 & 5,6 \\
2 & Teoría del Proceso & El Llano & Diurno & 5,3 & 4,7 & 6,3 & 6,2 & 5,5 \\
3 & Redacción Forense & Providencia & Vespertino & 5,3 & 5,5 & 6,4 & 4,8 & 5,7 \\
4 & $\begin{array}{l}\text { Derechos y Garantías } \\
\text { Constitucionales }\end{array}$ & Providencia & Diurno & 4,2 & 4,4 & 6,3 & 6,3 & 5,2 \\
\hline 5 & $\begin{array}{l}\text { Fuentes de las } \\
\text { Obligaciones }\end{array}$ & Providencia & Diurno & 5,6 & 5,5 & 5,7 & 5,6 & 5,6 \\
6 & $\begin{array}{l}\text { Derecho Civil IV } \\
\text { Totales }\end{array}$ & Providencia & Diurno & 5,1 & 5,9 & 6,1 & 6 & 5,8 \\
\hline
\end{tabular}

De lo anterior, es posible destacar la vinculación que hay entre la clase y el ejercicio de la profesión, que es lo que se busca con la metodología empleada. Ahora bien, no es solo una puesta en práctica de casos, sino que se debe seguir una metodología afín, con instrumentos de evaluación, con retroalimentación y una gran entrega de parte de los profesores para el logro de los aprendizajes de los estudiantes.

\section{Resultado en las evaluaciones}

En la tabla 5 y en la tabla 6, se evidencian los resultados de las evaluaciones en las asignaturas donde se utilizó la metodología propuesta.

Desde el punto de vista de los resultados del desempeño de los estudiantes, la propuesta metodológica fue todo un éxito.

Se evidencia una mejora significativa en la nota 3 , que corresponde al producto de aprendizaje evaluado en el proyecto. Esto ocurre en todas las asignaturas. En los últimos años, bajaba el rendimiento de los estudiantes por falta de interés en los últimos meses de clases, lo que se evidenciaba, además, en la asistencia (eventualmente por no haber examen) o, en algunos casos, por cierta falta de madurez solo esperaban una calificación que les permitiera aprobar sumando las dos primeras evaluaciones. Sin embargo, en los cursos evaluados a partir del proyecto ocurrió todo lo contrario, ya que la tercera evaluación fue la que obtuvo mejor rendimiento académico y fue la que más hizo trabajar a los estudiantes, tanto en horas presenciales como en trabajo autónomo fuera de aula.

Además, destaca que solo reprobaron estudiantes que se retiraron o desertaron de la carrera. Así, solo hubo siete personas reprobadas del total de 213, que son casos evidentes de retiro y/o deserción académica, lo que escapa de la propuesta metodológica empleada. 
Tabla 6. Consolidado del desempeño y de evaluaciones de las asignaturas del proyecto, calificación en aprobación o reprobación

\begin{tabular}{|lllllll|}
\hline Núm. & Asignatura & Campus & Jornada & Total de inscritos & Aprobados & Reprobados \\
\hline 1 & Teoría del Proceso & El Llano & Vespertino & 55 & 53 & 2 \\
\hline 2 & Teoría del Proceso & El Llano & Diurno & 49 & 46 & 3 \\
\hline 3 & $\begin{array}{l}\text { Redacción Forense } \\
4\end{array}$ & $\begin{array}{l}\text { Providencia } \\
\text { Derechos y Garantías }\end{array}$ & Vespertino & 19 & 19 & 0 \\
\hline 5 & $\begin{array}{l}\text { Fuentes de las Obliga- } \\
\text { ciones }\end{array}$ & Providencia & Diurno & 44 & 42 & 2 \\
\hline 6 & $\begin{array}{l}\text { Derecho Civil IV } \\
\text { Totales }\end{array}$ & Providencia & Diurno & 9 & 37 & 0 \\
* De las siete personas reprobadas, el promedio general de notas fue de 1,0 a 1,9, lo que evidencia casos de retiro o deserción \\
académica.
\end{tabular}

Y, desde el punto de vista del perfil de egreso y de los aprendizajes esperados/ resultados de aprendizaje, la propuesta metodológica contribuyó precisamente con ellos. Los estudiantes pudieron realizar un producto evidenciable que se utiliza en abogacía y que es parte del perfil de egreso. Elaboraron satisfactoriamente demandas, contestaciones, testamentos, escrituras de mandatos y recursos de protección, entre otros. Finalmente, también se situó a los estudiantes en problemas de la vida profesional para que los solucionen como lo haría un abogado. Esto, con la entrega de casos de acuerdo con la asignatura que se impartía.

\section{Conclusiones}

En los últimos años, ha habido una especial preocupación por el desempeño práctico de los futuros abogados. Esto ha sido advertido por tribunales, órganos del Estado y universidades. Por ello, se ha elaborado una propuesta metodológica que mejore el desempeño de los estudiantes, con énfasis en la actividad de la abogacía, para ser aplicada tanto en asignaturas teóricas como en las prácticas de la carrera de Derecho. En efecto, los resultados de la investigación han dado una comprobación en sentido positivo, sustentados con información objetiva relacionada con el desempeño de los estudiantes, como también de aquella proveniente de los actores clave en el proceso, es decir, de la percepción de los estudiantes y profesores.

Así, los resultados han evidenciado que sí es posible aplicar la propuesta metodológica, tanto en asignaturas teóricas y prácticas, y que trae como consecuencia una mejora de una serie de desempeños e indicadores, como son la progresión en los estudios; las competencias para elaborar productos relacionados con lo teórico de cada asignatura; el aumento de percepción de vinculación del curso con la profesión; 
la mejora de la evaluación docente; la motivación y la confianza de los estudiantes; y la evidencia de trabajo autónomo efectivo; entre otros aspectos positivos.

También se comprobó que es posible aplicar la metodología en asignaturas de derecho sustantivo, de público y privado, y de derecho adjetivo, como procesal, no habiendo ninguna diferencia significativa. Al mismo tiempo, no hay contrastes de relevancia entre campus y jornadas diurnas o vespertinas en la aplicación de la propuesta. Más bien, existen discordancias con las metodologías tradicionales, de medición de conocimientos teóricos a través de pruebas tipo test, exámenes orales solemnes, es decir, de demostración de saberes más que de la aplicación de estos.

Es por ello que la hipótesis comprobada en los términos descritos implica que no solo es posible aplicar la propuesta metodológica, sino que se genera la necesidad de utilizarla en el quehacer docente de la carrera. Esto, sustentado en información de percepción académica y en datos objetivos cuantificables. Es relevante, por ejemplo, que aprobaron cada asignatura 206 estudiantes de 213, siendo los 7 reprobados casos evidentes de retiro o recesos académicos y, especialmente, la mejora significativa del rendimiento individual, de regular a sobresaliente en todos los cursos en la propuesta académica, evidenciado con productos propios de la abogacía.

\section{Referencias}

Barrios Araya, Silvia, Miriam Rubio Acuña, Marina Gutiérrez Núñez y Carola Sepúlveda Vería (2012). «Aprendizaje-servicio como metodología para el desarrollo del pensamiento crítico en educación superior». Revista Cubana de Educación Médica Superior, 26 (4): 594-603. Disponible en bit.ly/3yuwyrn.

Beca Frei, Juan Pablo, Eduardo Guillermo Castillo Vigouroux, Alejandra Cid Droppelmann, Eduardo Alberto Darritchon Pool y Scarlette María Loreto Lagos Fuentes (2019). «Diseño de un examen de grado por competencias en la carrera de Derecho». Revista Pedagogía Universitaria y Didáctica del Derecho, 6 (1): 99-128. DOI: 10.5354/0719-5885.2019. 53747.

BlázQuez Martin, Diego (2015). «Clínicas de derechos humanos: Causas y ventajas del éxito de un modelo de educación jurídica del siglo XXI». Revista de Educación $y$ Derecho, 11: 1-24. Disponible en bit.ly/3fktQNB.

Bonet SÁnchez, María Pilar (2018a). «Clínicas jurídicas. Clínica de atención directa». En Ruth M. Mestre i Mestre (coordinadora), Guía práctica para la enseñanza del derecho a través de las clínicas jurídicas. Diez años de experiencia de la Clínica Jurídica per la Justicia Social de la Facultad de Dret de la Universitat de Valéncia (pp. 23-29). Valencia: Tirant lo Blanch.

-. (2018b). «Fichas técnicas. Clínica de atención directa». En Ruth M. Mestre i Mestre (coordinadora), Guía práctica para la enseñanza del derecho a través de las clínicas jurídicas. Diez años de experiencia de la Clínica Jurídica per la Justicia Social 
de la Facultad de Dret de la Universitat de Valéncia (pp. 63-66). Valencia: Tirant lo Blanch.

Camilloni, Alicia (2019). «La enseñanza del derecho orientada al desarrollo de la creatividad». Revista Pedagogía Universitaria y Didáctica del Derecho, 6 (1): 5-22. DOI: $10.5354 / 0719-5885.2019 .53743$.

Comisión NaCional de ACReditación (CAN) (2020). CRITERIOS DE EVALUACióN PARA CARRERAS DE DERECHO.

De la Peña Amorós, María del Mar y Marta Marcos Cardona (2010). «La clase magistral como metodología docente adaptable al EEES». En Eva María Rubio, María Magnolia Pardo y Mercedes Farias (coordinadoras), Buenas prácticas para la docencia del Derecho adaptada al Ects (pp. 31-40). Murcia: Universidad de Murcia.

De Miranda Vésquez, Carlos (2017). «La didáctica del derecho orientada al «saber hacer». En Carlos Espaliú Berbud, Ricardo María Jiménez-Yáñez y Carlos De Miranda Vázquez (directores), ¿Cómo la innovación mejora la calidad de la enseñanza del derecho? Propuestas en un mundo global (pp. 83-95). Navarra: Thomson Reuters.

Elgueta Rosas, María Francisca y Eric Palma González (2014). «Una propuesta de clasificación de la clase magistral impartida en la Facultad de Derecho». Revista chilena de derecho, 41 (3): 907-924. DOI: 10.4067/So718-34372014000300006.

Espinoza Freire, Eudaldo Enrique (2018). «La hipótesis en la investigación». Mendive, 16 (1): 122-139. Disponible en bit.ly/3vcCW4l.

Gabilondo, José (2017). «El método de los casos en la pedagogía jurídica de los EEUU: Una valoración subjetiva». Revista Pedagogía Universitaria y Didáctica del Derecho, 4 (2): 74-114. DOI: 10.5354/0719-5885.2017.46251.

GiL-Galván, Rosario (2018). «El uso del aprendizaje basado en problemas en la enseñanza universitaria. Análisis de las competencias adquiridas y su impacto». Revista mexicana de investigación educativa, 23 (76): 73-93. Disponible en bit. ly/3hKnuZB.

Gómez Francisco, Taeli y Juan Rubio González (2017). «Complejidad-educación: Un aporte para las generaciones futuras». Revista Educación y Humanismo, 19 (33): 409-424. DOI: 10.17081/eduhum.19.33.2653.

Hernández Sampieri, Roberto, Carlos Fernández Collado y Pilar Baptista Lucio (2013). Metodología de la Investigación. México: Mcgraw-Hill.

Manterola, Carlos y Tamara Otzen (2013). «Porqué investigar y cómo conducir una investigación». International Journal of Morphology, 31 (4): 1498-1504. DOI: 10.4067/So717-95022013000400056.

Martínez Girón, Jesús y Alberto Arufe Varella (2015). La enseñanza del derecho en las facultades de Derecho de los Estados Unidos: A propósito de la enseñanza en ellas del derecho del trabajo y del derecho de la seguridad social. Barcelona: Atelier. 
Matus Acuña, Jean Pierre (2007). «Control ético y deontología». Ius et Praxis, 13 (1): 463-472. DOI: 10.4067/S0718-00122007000100016.

Orihuela Calatayud, Esperanza y Eva María Rubio Fernández (2010). «La clínica jurídica como metodología: Una propuesta para la renovación». En Eva María Rubio, María Magnolia Pardo y Mercedes Farias (coordinadoras), Buenas prácticas para la docencia del derecho adaptada al Ects (pp. 143-173). Murcia: Universidad de Murcia.

Ramírez Ortiz, Patricia e Ignacio Luna Espinoza (2017). «Aprendizaje basado em problemas: metodología didáctica para abordar la asignatura de Derecho Procesal Civil». Revista Pedagogía Universitaria y Didáctica del Derecho, 4 (1): 189-224. DOI: $10.5354 / 0719-5885.2017 .46254$.

Selma Penalva, Alejandra (2010). «Los casos prácticos como herramienta docente». En Eva María Rubio, María Magnolia Pardo y Mercedes Farias (coordinadoras), Buenas prácticas para la docencia del derecho adaptada al Ects (pp. 41-62). Murcia: Universidad de Murcia.

Solari, Enzo (2012). «El currículo chileno de estudios jurídicos». Revista de Derecho (Valdivia), 19 (2): 703-734. DOI: 10.4067/So718-68512012000200024.

Sologuren Insúa, Enrique, Cristina Bonifaz Reyes y Carmen Gloria Núñez Castillo (2019). «El curso basal de competencias comunicativas en derecho: Enseñanza de la escritura académica desde un enfoque mixto e interdisciplinar». Revista Pedagogía Universitaria y Didáctica del Derecho, 6 (1): 129-152. DOI: 10.5354/0719-5885.2019.53748.

SuAzo, Iván (2017). «El marco general del sistema de acreditación de las instituciones de educación superior en Chile». Revista de Educación y Derecho, 15: 1-5. Disponible en bit.ly/3fbkW4X.

Universidad Autónoma de Chile (2020). Malla curricular Derecho. Disponible en bit.ly/3vpwtTO.

Universidad Católica de Chile (2020). Licenciatura en Derecho. Disponible en bit.ly/3bMNsI 3 .

Universidad De Chile (2020). Malla curricular de la carrera de Derecho. bit. ly/3C58hif.

VALLÉs Rapp, Cristina, Lurdes Martínez Mínguez y María del Rosario Romero Martín (2018). «Instrumentos de evaluación: Uso y competencia del profesorado universitario en su aplicación». Estudios pedagógicos (Valdivia), 44 (2): 149-169. DOI: 10.4067/So718-07052018000200149. 


\section{Agradecimientos}

Este trabajo recoge los resultados del proyecto titulado «Elaboración de instrumentos de evaluación centrados en el estudiante de la carrera de Derecho de la Universidad Autónoma de Chile (DDPo3-2018)», proveniente del concurso realizado por la Universidad Autónoma de Chile, adjudicado e implementado.

\section{Sobre el autor}

Juan Pablo Díaz Fuenzalida es doctor en Derecho y máster en Gobernanza y Derechos Humanos, ambos por la Universidad Autónoma de Madrid. Además, es abogado, licenciado en Ciencias Jurídicas y Sociales, y magíster en Docencia Universitaria, ambos por la Universidad Autónoma de Chile. Es profesor de Derecho adscrito al Instituto de Investigación en Derecho de la Universidad Autónoma de Chile e Investigador responsable del proyecto Fondecyt de Postdoctorado 2020 (3200477) titulado «Instituto Nacional de Derechos Humanos (INDH): Evaluación de sus 10 años de protección y promoción de los derechos humanos en los tribunales de justicia en Chile». Sus correos electrónicos son jpdiazfuenzalida@gmail.com y juanpablo. diaz@uautonoma.cl. (D) https://orcid.org/oooo-0002-6490-9542. 
La Revista Pedagogía Universitaria y Didáctica del Derecho (RPUDD) es una publicación científica semestral que contribuye a la reflexión multidisciplinaria sobre pedagogía universitaria y didáctica del derecho, para la formación y consolidación de esta área de investigación; así como a la difusión de prácticas innovadoras en la enseñanza-aprendizaje del derecho considerando el contexto nacional e internacional. Es una publicación electrónica internacional con una codirección entre Brasil y Chile.

\author{
DIRECTORA \\ María Francisca Elgueta Rosas \\ Universidad de Chile \\ DIRECTOR \\ Renato Duro Dias \\ Universidad Federal de Rio Grande, Brasil \\ SITIO WEB \\ pedagogiaderecho.uchile.cl \\ CORREO ELECTRÓNICO \\ rpedagogia@derecho.uchile.cl \\ LICENCIA DE ESTE ARTÍCULO
}

Creative Commons Atribución Compartir Igual 4.o Internacional

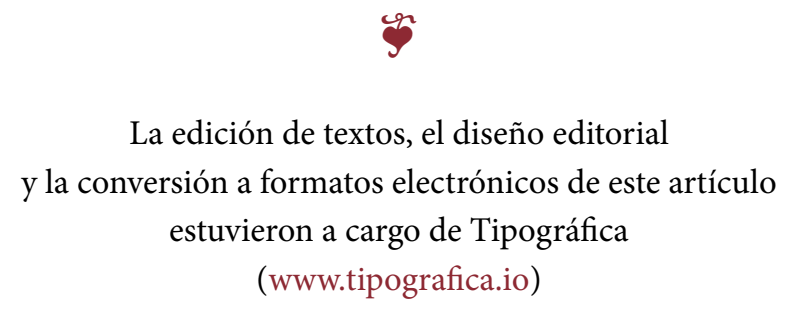

